\title{
The associations between coronary artery disease, and non-alcoholic fatty liver disease by computed tomography
}

Samira Saraya², Mahmoud Saraya ${ }^{1^{*}} \mathbb{0}$, Mohamed Mahmoud ${ }^{3}$, Mohamed Galal $^{4}$, Hazem Hamed Soliman ${ }^{2}$ and Mariam Raafat ${ }^{2}$

\begin{abstract}
Background: Non-alcoholic fatty liver disease (NAFLD) is increasing in recognition as a hepatic condition that is unrelated to significant alcoholic consumption, but has rather, been suggested to constitute cardiovascular risk (irrespective of traditional risk factors and high-risk plaque features). Both coronary artery disease and NAFLD share the same pathophysiology and metabolic profile. NAFLD can theoretically be a source/initiator for coronary artery disease (CAD). We aimed to study the association between NAFLD, CAD, the presence of high-risk plaque features, and the severity of stenosis.
\end{abstract}

Results: We recruited 800 patients with suspected obstructive CAD and planned for coronary computed tomography angiography (CCTA), Exclusion criteria: heavy alcohol consumption; contraindications to contrast media; unevaluated coronary-artery segments; other known liver disease; and use of oral corticosteroids and/or amiodarone. Non-enhanced Computed Tomography abdomen was performed before the CCTA to detect NAFLD. To study the association between NAFLD and the presence of CAD, patients were classified as to either have, or not have CAD. The CAD group were then further studied for the presence of high-risk plaque features: napkin ring sign, Positive remodelling, Low Hounsfield unit (HU), and Spotty calcium; and their association with NAFLD. Thirty-two per cent of patients had NAFLD and 45\% had CAD. A significant association between NAFLD and CAD was found (OR 4.21, 95\% $\mathrm{Cl}$ (confidence interval) (2.83-6.25), $p=0.000)$. In CAD patients, significant associations were present between NAFLD and high-risk plaque features: Napkin ring sign, Positive remodelling, Low $\mathrm{HU}$, and Spotty calcium $(\mathrm{OR} 7.88,95 \% \mathrm{Cl}$ (4.39-14.12), $p<0.001, \mathrm{OR} 5.84,95 \%$ (3.85-8.85), $p<0.001, \mathrm{OR} 7.25,95 \% \mathrm{Cl}(3.31-15.90), p<0.001$ and OR 6.66, 95\% Cl $(3.75-11.82), p<0.001)$, respectively. NAFLD was present in $39.30 \%, 50.00 \%, 20.00 \%, 54.50 \%$ and $100.00 \%$ of patients with CAD; and 1-24\%; 25-49\%; 50-69\%; 7 =0-99\%, LMD (Left Main Disease) $>50 \%$ stenosis or 3V disease, and Total occlusion, respectively, $p<0.001$.

Conclusions: NAFLD is strongly associated with CAD, high-risk plaque features and higher grade of stenosis.

Keywords: Computed tomography, Coronary artery disease, Non-alcoholic fatty liver disease

\footnotetext{
*Correspondence: msaraya@kasralainy.edu.eg; msaraya@cu.edu.eg

${ }^{1}$ Cardiovascular Department, Faculty of Medicine, Cairo University, $12 \mathrm{Al}$

Saraya Street, El Manial, Cairo P.O 11555, Egypt

Full list of author information is available at the end of the article
}

\begin{abstract}
Background
Non-Non-Alcoholic Fatty Liver Disease (NAFLD) is an increasingly recognized hepatic condition that encompasses a wide spectrum of liver affection ranging from simple steatosis to advanced fibrosis, cirrhosis, and hepatocellular carcinoma. It is not essentially related to significant alcohol intake $[1,2]$.
\end{abstract}

\section{Springer Open}

(c) The Author(s) 2021. Open Access This article is licensed under a Creative Commons Attribution 4.0 International License, which permits use, sharing, adaptation, distribution and reproduction in any medium or format, as long as you give appropriate credit to the original author(s) and the source, provide a link to the Creative Commons licence, and indicate if changes were made. The images or other third party material in this article are included in the article's Creative Commons licence, unless indicated otherwise in a credit line to the material. If material is not included in the article's Creative Commons licence and your intended use is not permitted by statutory regulation or exceeds the permitted use, you will need to obtain permission directly from the copyright holder. To view a copy of this licence, visit http://creativecommons.org/licenses/by/4.0/. 
Variation in the prevalence of NAFLD is found based on factors such as race, ethnicity, age, gender, geographical distribution, and the diagnostic modality used [3-5]. NAFLD has been firmly linked to insulin resistance, type 2 diabetes mellitus, dyslipidaemia, and metabolic syndrome, especially in patients with sedentary lifestyles, changing dietary patterns and increased obesity $[2,6]$. These factors also comprise the risk profile for coronary artery disease (CAD). Both CAD and NAFLD share the same underlying pathophysiological mechanism, risk factors, lifestyle modification and treatment plans, and were therefore hypothesized to be closely related [7].

NAFLD is recently suggested to be an independent risk for cardiovascular diseases and is also associated with higher cardiovascular mortality [8].

Recent advances in the coronary CT (computed tomography) made it possible to diagnose obstructive CAD in acute and chronic conditions, with identification of high-risk plaque (positive remodelling, low CT attenuation, spotty calcium, or napkin ring sign). The presence of high-risk plaque was associated with a significantly increased risk of major adverse cardiac events [9]. Furthermore, non-enhanced abdominal CT has been found to have a quantitative assessment for steatosis with good sensitivity and specificity [10].

Based on these findings, we aimed to study the association between NAFLD, the obstructive CAD, the presence of high-risk coronary plaque as depicted by $\mathrm{CT}$, and the severity of stenosis in an Egyptian cohort.

\section{Methods}

Eight hundred patients with clinically suspected CAD referred to the radiology department for Coronary Computed Tomography Angiography (CCTA) were enrolled. Demographic data and medical history with special emphasis on cardiovascular risk factors (systemic arterial hypertension, diabetes mellitus, dyslipidaemia, and smoking status) were collected from all subjects. The study protocol conformed to the ethical guidelines of the 1975 Declaration of Helsinki and was approved by the Institutional Review Board of Radiology. A written informed consent was obtained from all participants before enrolling.

\section{Exclusion criteria}

Patients presented with any of the following factors were excluded: (1) history of consumption of more than $20 \mathrm{~g}$ of alcohol per day; (2) contraindications to contrast media "e.g. history of reactions to any of the contrast agents, pregnancy, treatment of thyroid disease with radioactive iodine, and chronic or acutely worsening renal disease"; (3) unevaluated coronary-artery segments owing to motion artefacts or inadequate contrast medium filling;
(4) other known liver diseases (carriers of the hepatitis B virus or hepatitis $C$ virus); (5) use of oral corticosteroids and/or amiodarone that may be implicated in causing fatty liver at test time.

\section{CT assessment of NAFLD}

Two senior radiologists with over ten years of experience in abdominal CT (M.R. and M.M.) were blinded to the results of CCTA and performed measurements of hepatic and splenic $\mathrm{CT}$ attenuation on the non-enhanced $\mathrm{CT}$ scans independently.

The hepatic CT attenuation was measured by obtaining the mean of the attenuation values for three circular regions of interest with an area of at least $2 \mathrm{~cm}^{2}$ that were placed at three different hepatic levels. Areas of hepatic vascular and biliary structures were avoided. The splenic $\mathrm{CT}$ attenuation was calculated similarly.

NAFLD was defined as either hepatic CT attenuation minus splenic CT attenuation of less than $1 \mathrm{HU}$ (Hounsfield unit), or the mean $\mathrm{CT}$ number ratio of liver-tospleen parenchyma of less than $1 \mathrm{HU}[11,12]$.

\section{CCTA analysis and high-risk coronary plaque assessment} Conventional CCTA was performed using a 64-detector row scanner (Toshiba, Aquilion TSX-101A). The images were then analysed and evaluated by two experienced senior radiologists (S.S and H.H.). The protocol followed the Society of Cardiovascular CT guidelines, which started by calculation of calcium score employing nonenhanced CT followed by contrast enhanced CCTA [13]. Coronary segments were then evaluated for the presence of coronary atherosclerotic plaque and the presence stenosis. The severity of the stenosis was categorized based on Coronary Artery Disease Reporting and Data System (CAD-RADS) classification [14].

The presence of any high-risk plaque feature such as positive remodelling, spotty calcium, napkin ring sign or low CT number was reported blindly to the presence/ absence of NAFLD [15].

\section{Patients categorization and classification}

Patients were classified into two groups; the first group were those who were diagnosed with CAD (CAD-RADS 1 or more), and the second group were those who had no CAD (CAD-RADS 0 ) as shown by CCTA.

Subgroup analysis of the first group regarding the severity of stenosis and the presence of high-risk plaque features was then performed.

\section{Results}

\section{Statistical methods}

Microsoft excel 2013 was used for data entry, and the SPSS (statistical package for social science) version 24 
was used for data analysis. All collected inquiries were revised for competences and logical consistency.

Simple descriptive statistics (arithmetic mean and standard deviation) was used for the summary of normal quantitative data and frequencies used for qualitative data. The bivariate relationship was displayed in cross-tabulations, and comparison of proportions was performed using the Chi-square and Fisher's exact tests, where appropriate. Independent T-test, oneway ANOVA (ANalysis of Variance) and post hoc tests were used to compare normally distributed quantitative data. The level of significance was set at probability (P) value $<0.05$. The factors that were significantly associated with CAD and NAFLD in bivariate analysis $(p<0.05)$ were included in multivariate logistic regression models.

\section{Patients' characteristics}

The ages of our study population ranged from 21 to 79 years with a mean of $52.46 \pm 11.02$ years, majority were males, and NAFLD was the most frequent clinical

Table 1 Patients' characteristics

\begin{tabular}{lll}
\hline & $\boldsymbol{n}$ & (\%) \\
\hline Male gender & 568 & $71 \%$ \\
NAFLD & 256 & $32 \%$ \\
Htn & 232 & $29 \%$ \\
DM & 168 & $21 \%$ \\
Smoking & 96 & $12 \%$ \\
Dyslipidaemia & 174 & $21.8 \%$ \\
Calcium score (AU) & & \\
0 & 424 & $53 \%$ \\
$1-100$ & 296 & $37 \%$ \\
$101-300$ & 48 & $6 \%$ \\
$>300$ & 32 & $4 \%$ \\
\hline AU,Agatston units; DM, diabetesmelitus;
\end{tabular}

$\mathrm{AU}$, Agatston units; DM, diabetes mellitus; $\mathrm{Htn}$., Hypertension risk factor in the population. Ten per cent of our study subjects had calcium scores of more than 100. The frequencies of clinical risk factors for CAD are demonstrated in Table 1. Examples for patients demonstrating NAFLD and high-risk plaque features of the coronaries are depicted in Figs. 1, 2, 3, 4.

\section{Comparison between patients with CAD and non-CAD}

CAD was present in 440 (55\%) subjects among the entire study group. Their demographics and risk factors of both groups are outlined in Table 2.

\section{Regression analysis for the presence of CAD}

Direct logistic regression was performed to assess the impact of several factors on the likelihood that patients would have CAD. The model contained seven independent variables: age, sex, NAFLD, Htn. (Hypertension), DM (Diabetes Mellitus), smoking \& dyslipidaemia. The full model containing all factors was statistically significant, $\mathrm{X} 2(7,800)=185.14, p$ value $<0.001$, indicating that the model was able to distinguish between patients with and without CAD. The model explained between 20.7\% (Cox and Snell R square) and 27.6\% (NagelKerke R squared) of the variance in CAD status and correctly classified $70.8 \%$ of cases. As shown in Table 3, only four of the independent variables made a unique statistically significant contribution to the model (age, sex, NAFLD \& smoking). The strongest independent predictor of having CAD was NAFLD, recording an odds ratio (OR) of 4.21 and controlled for all other factors in the model.

\section{Subgroup analysis of CAD patients}

Approximately $25 \%$ of patients had significant CAD as shown by CCTA, with more than $70 \%$ coronary artery narrowing. Table 4 Calcified and noncalcified plaques were strongly associated with NAFLD among patients

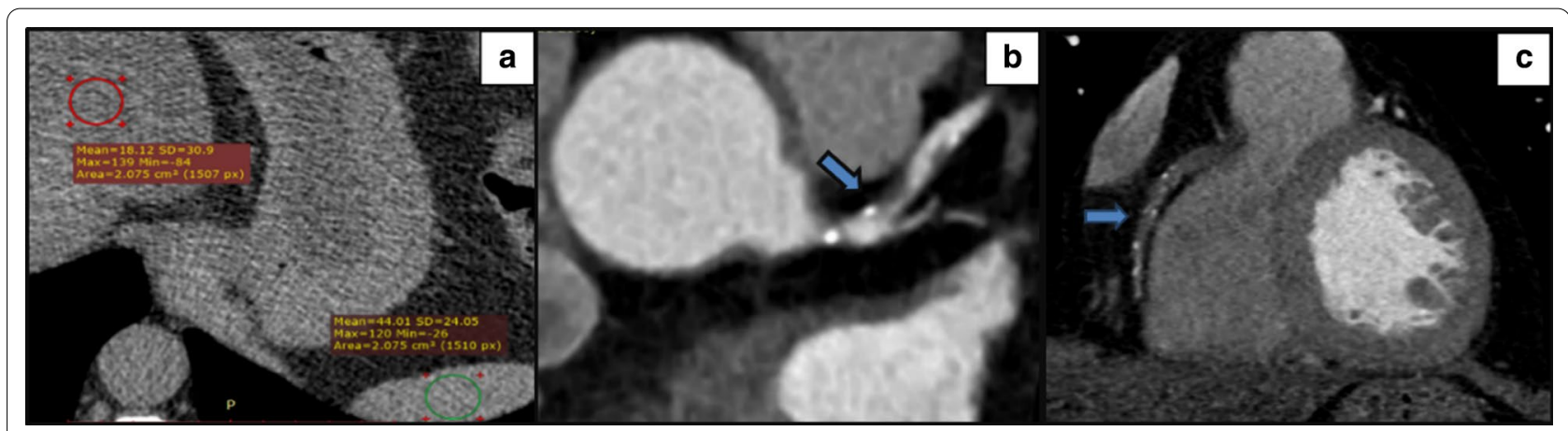

Fig. 1 Hypertensive male patient 55 years old. a Axial non-enhanced CT image demonstrates attenuation measurements of the liver and spleen showing fat accumulation in the liver, with a mean liver attenuation of $18.1 \mathrm{HU}$ and a mean spleen attenuation of $44 \mathrm{HU}$. $\mathbf{b}$ Coronary CT angiography shows partially calcified plaque with a low CT number (17 HU) (arrow) and positive remodelling and c Coronary CT angiography shows totally occluded RCA with multiple spotty calcifications (arrow) 


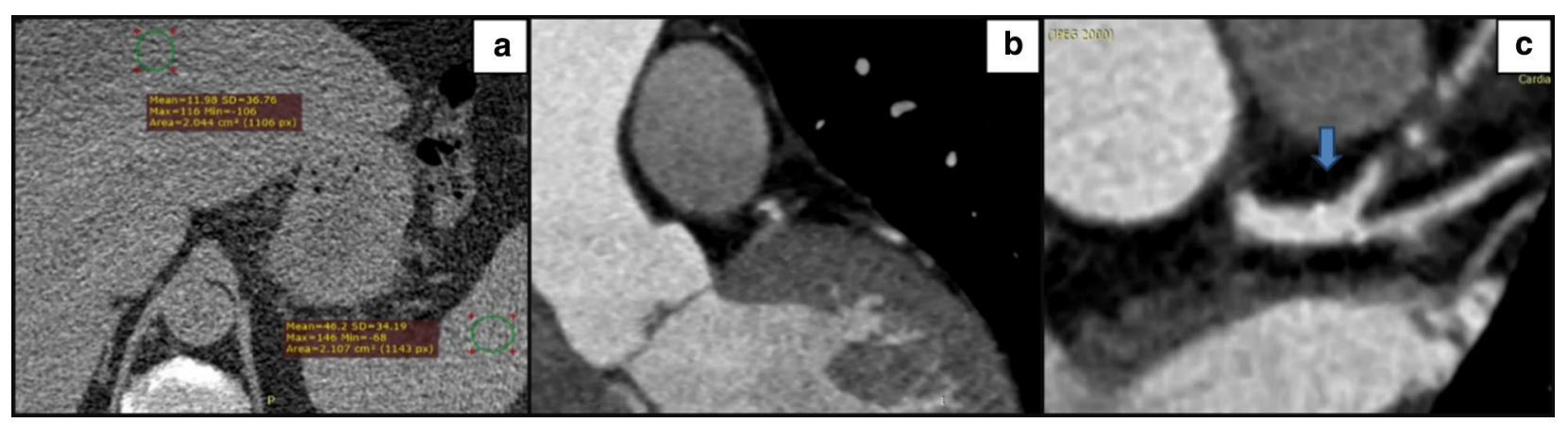

Fig. 2 A male patient 67 years old, hypertensive, diabetic, and smoker. a Axial non-enhanced $C T$ demonstrates attenuation measurements of the liver and spleen with a mean liver attenuation of $11.9 \mathrm{HU}$ and a mean spleen attenuation of $46.2 \mathrm{HU}$. b Coronary CT angiography images shows a non-calcified plaque demonstrating a napkin-ring sign with a central low-attenuation area, surrounded by a peripheral rim of higher attenuation. $\mathbf{c}$ Coronary CT angiography images shows a calcified ostial lesion with spotty calcifications (arrow) of left main coronary artery

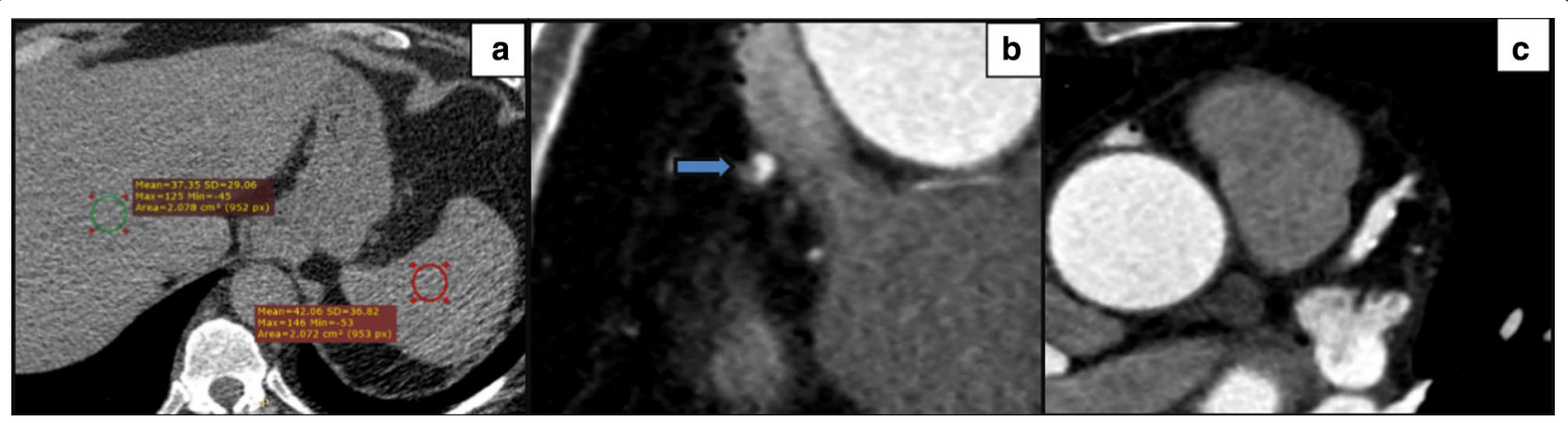

Fig. 3 Diabetic male patient 67 years old. a Axial non-enhanced CT image demonstrates attenuation measurements of the liver and spleen shows a diffuse fat accumulation in the liver, with a mean liver attenuation of $37.3 \mathrm{HU}$ and a mean spleen attenuation of $42.5 \mathrm{HU}$. $\mathbf{b}$ Coronary CT angiography images shows a non-calcified plaque demonstrates a napkin-ring sign (arrow) with a central low-attenuation area, surrounded by a peripheral rim of higher attenuation and c Coronary CT angiography images shows a plaque with spotty calcium

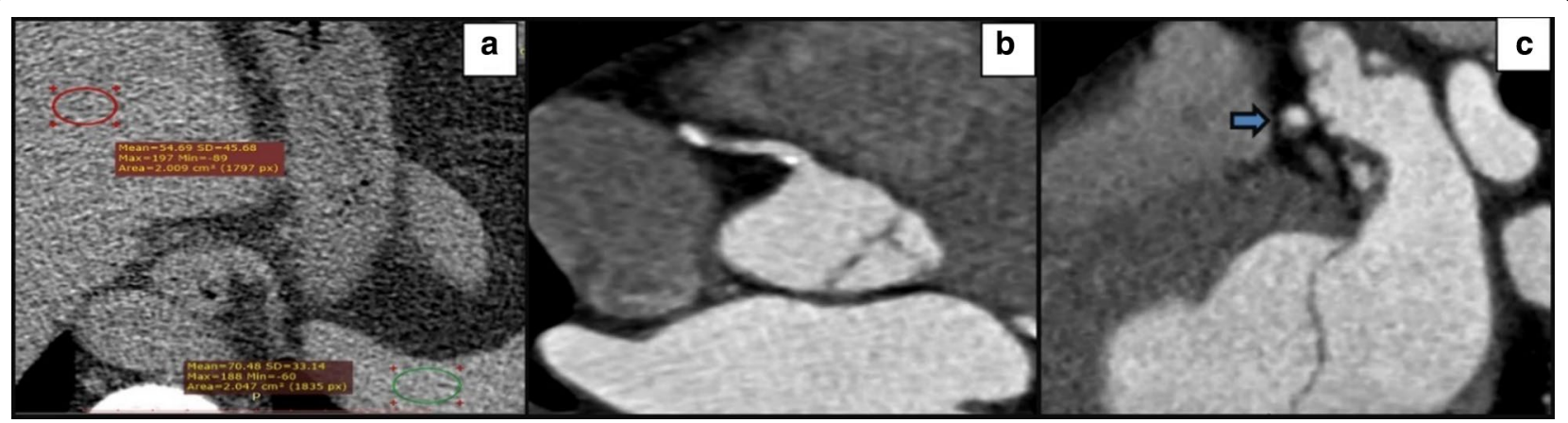

Fig. 4 A 65-year-old lady with diabetes shows: a Axial non-enhanced CT image demonstrates attenuation measurements of the liver and spleen shows a diffuse fat accumulation in the liver, with a mean liver attenuation of $54.6 \mathrm{HU}$ and a mean spleen attenuation of $70.4 \mathrm{HU}$. b Coronary CT angiography images shows a plaque with spotty calcium and c Coronary CT angiography shows a non-calcified plaque demonstrates a napkin-ring sign(arrow) with a central low-attenuation area, surrounded by a peripheral rim of higher attenuation

with CAD (OR 12, 95\% (Confidence Interval) CI 5.632$25.568, p$ value $<0.001$, OR, 95\% CI 2.315, 1.571-1.067, $p$ value 0.022 ).
High-risk plaque features (napkin ring sign, positive remodelling, low $\mathrm{HU}$, and spotty calcium) were studied among the different clinical risk factors. NAFLD 
Table 2 Comparison between CAD and non-CAD patients

\begin{tabular}{lllr}
\hline & $\begin{array}{l}\text { CAD }(\boldsymbol{n} \text { 440) } \\
\boldsymbol{n}(\%)\end{array}$ & $\begin{array}{l}\text { Non-CAD }(\boldsymbol{n} \text { 360) } \\
\boldsymbol{n}(\%)\end{array}$ & $\boldsymbol{p}$ value \\
& Mean ( \pm SD) & Mean $( \pm$ SD) & \\
\hline Age (years) & $55.28 \pm 10.19$ & $49.01 \pm 11.031$ & $<0.001$ \\
Male Gender & $328(74.5 \%)$ & $240(66.7 \%)$ & 0.015 \\
NAFLD & $200(45.5 \%)$ & $56(15.6 \%)$ & $<0.001$ \\
Htn & $144(32.7 \%)$ & $88(24.4 \%)$ & 0.01 \\
DM & $120(27.3 \%)$ & $48(13.3 \%)$ & $<0.001$ \\
Smoking & $64(14.5 \%)$ & $32(8.9 \%)$ & 0.014 \\
Dyslipidaemia & $126(28.6 \%)$ & $48(13.3 \%)$ & $<0.001$ \\
\hline
\end{tabular}

CAD, coronary artery disease; DM, diabetes mellitus; Htn., Hypertension; NAFLD, non-alcoholic fatty liver disease

Table 3 Multivariate logistic regression predicting the likelihood of reporting CAD:

\begin{tabular}{lll}
\hline & OR $\mathbf{( 9 5 \% ~ C l )}$ & $\boldsymbol{p}$ value \\
\hline Age (years) & $1.08(1.06-1.1)$ & 0.000 \\
Male Gender & $1.69(1.18-2.41)$ & 0.004 \\
NAFLD & $4.21(2.83-6.25)$ & 0.000 \\
Htn & $0.68(0.46-1.02)$ & 0.059 \\
DM & $2.24(0.92-5.13)$ & 0.055 \\
Smoking & $3.07(1.76-5.37)$ & 0.000 \\
Dyslipidemia & $0.81(0.36-1.85)$ & 0.616
\end{tabular}

DM, diabetes mellitus; Htn., Hypertension; NAFLD, non-alcoholic fatty liver disease

Table 4 Percent stenosis and plaque characteristics in patients with CAD

\begin{tabular}{lll}
\hline & $\mathbf{n}$ & $\%$ \\
\hline Percent coronary stenosis & & \\
$1-24 \%$ stenosis & 224 & $50.9 \%$ \\
$25-49 \%$ stenosis & 64 & $14.5 \%$ \\
$50-69 \%$ stenosis & 40 & $9.1 \%$ \\
$70-99 \%$ stenosis-10r 2 vessel disease & 64 & $14.5 \%$ \\
LMD $>$ 50\%-3VD & 24 & $5.5 \%$ \\
Total occlusion & 24 & $5.5 \%$ \\
Calcified plaques & 352 & $80 \%$ \\
Non-calcified plaques & 168 & $38 \%$ \\
High-risk features & & \\
Napkin ring sign & 88 & $20 \%$ \\
Positive remodelling & 184 & $42 \%$ \\
Low HU & 48 & $11 \%$ \\
Spotty calcium & 336 & $76 \%$ \\
\hline
\end{tabular}

$3 V D$, three vessel disease; $C A D$, Coronary artery disease; HU, Hounsfield unit; LMD, left main disease was found to be associated with an increased likelihood of having high-risk features among patients with CAD (Table 5).

Compared to patients without napkin ring sign, patients with Napkin ring sign were significantly older $(57.9 \pm 7.4$ vs $54.63 \pm 10.7$ years, $p$ value 0.001 ). This finding was not found with other CAD high-risk features including positive remodelling $(54.6 \pm 9.8$ vs $55.8 \pm 10.4$ years, $p$ value $0.2)$, low $\mathrm{HU}(55.8 \pm 7.6$ vs $55.2 \pm 10.47$ years, $p$ value $0.608)$ and spotty calcium ( $55.4 \pm 9.8$ vs $55.1 \pm 11.4$ years, $p$ value 0.793$)$, There was no association found between the presence of NAFLD and a high calcium score of over 100 AU (Agatston Units) (OR 0.76, 95\% CI 0.46-1.25, $p$ value 0.279 ).

There was a significant difference among groups with different severity of stenosis, and NAFLD was present in $39.30 \%, 50.00 \%, 20.00 \%, 54.50 \%$ and $100.00 \%$ in patients with CAD and 1-24\%, 25-49\%, 50-69\%, (70-99\% stenosis, LMD (Left Main Disease) $>50 \%$ or $3 \mathrm{~V}$ disease), and Total occlusion, respectively, $p<0.001$ (Fig. 5).

\section{Discussion}

Recent increased awareness of the NAFLD deemed essential as it has been shown to correlate with other diseases, in particular atherosclerotic CAD [16, 17]. It has been suggested that NAFLD induces a systemic inflammatory response, increased oxidative stress, insulin resistance, fatty acid toxicity and endothelial dysfunction; all of which share the same pathophysiology for development of atherosclerotic CAD [3, 18, 19].

Despite being currently considered one of the leading liver diseases with an estimated prevalence of $15 \%-40 \%$ in the general population $[3,20]$, high variations in NAFLD prevalence are observed among different populations owing to several factors such as socio-economic status, ethnicity, the method of diagnosis, and reporting of the disease [7, 21].

NAFLD is usually defined as a deposition of lipid in more than $5 \%$ of the liver in the absence of enduring or recent heavy alcohol consumption ( $>20 \mathrm{gm} /$ day), and after exclusion of other drugs and medical conditions that can enhance this phenomenon [22].

The prevalence of NAFLD in our study population was $32 \%$, where the study cohort showed a male gender prevalence representing $71 \%$ of patients.

Our study is considered the largest in Egypt to examine the association between NAFLD and CAD, as it involved 800 patients. We found that there is not only a statistically significant association between CAD had NAFLD, but NAFLD was also significantly associated with the presence of a high-risk plaque feature and the grade of stenosis in patients diagnosed with CAD as detected by the CCTA. We used the CT modality unenhanced and 
Table 5 Adjusted Prevalence odd's ratio of high-risk plaque features according to clinical risk factors (Male gender, NAFLD, Htn., DM, smoking and Dyslipidaemia) in 440 patients with CT findings of coronary artery disease

\begin{tabular}{|c|c|c|c|c|c|c|c|c|}
\hline & \multicolumn{2}{|c|}{ Napkin ring sign (88) } & \multicolumn{2}{|c|}{$\begin{array}{l}\text { Positive remodelling } \\
\text { (184) }\end{array}$} & \multicolumn{2}{|c|}{ Low HU (48) } & \multicolumn{2}{|c|}{ Spotty calcium (336) } \\
\hline & $\begin{array}{l}\text { OR } \\
95 \% \mathrm{Cl}\end{array}$ & $p$ value & $\begin{array}{l}\text { OR } \\
95 \% \mathrm{Cl}\end{array}$ & $p$ value & $\begin{array}{l}\text { OR } \\
95 \% \mathrm{Cl}\end{array}$ & $p$ value & $\begin{array}{l}\text { OR } \\
95 \% \mathrm{Cl}\end{array}$ & $p$ value \\
\hline Males & $\begin{array}{l}1.69 \\
(0.94-3.05)\end{array}$ & 0.08 & $\begin{array}{l}2.16 \\
(1.36-3.43)\end{array}$ & 0.001 & $\begin{array}{l}0.854 \\
(0.82-0.89)\end{array}$ & $<0.001$ & $\begin{array}{l}3.64 \\
(2.27-5.84)\end{array}$ & $<0.001$ \\
\hline NAFLD & $\begin{array}{l}7.88 \\
(4.39-14.12)\end{array}$ & $<0.001$ & $\begin{array}{l}5.84 \\
(3.85-8.85)\end{array}$ & $<0.001$ & $\begin{array}{l}7.25 \\
(3.31-15.9)\end{array}$ & $<0.001$ & $\begin{array}{l}6.66 \\
(3.75-11.82)\end{array}$ & $<0.001$ \\
\hline $\mathrm{Htn}$ & $\begin{array}{l}1.99 \\
(1.23-3.21)\end{array}$ & 0.004 & $\begin{array}{l}0.59 \\
(0.39-0.89)\end{array}$ & 0.012 & $\begin{array}{l}2.267 \\
(1.24-4.15)\end{array}$ & 0.007 & $\begin{array}{l}0.72 \\
(0.45-1.13)\end{array}$ & 0.154 \\
\hline DM & $\begin{array}{l}1.71 \\
(1.04-2.82)\end{array}$ & 0.032 & $\begin{array}{l}1.31 \\
(0.86-2.00)\end{array}$ & 0.207 & $\begin{array}{l}1.385 \\
(0.73-2.63)\end{array}$ & 0.318 & $\begin{array}{l}2.47 \\
(1.38-4.40)\end{array}$ & 0.002 \\
\hline Smoking & $\begin{array}{l}0.53 \\
(0.24-1.15)\end{array}$ & 0.105 & $\begin{array}{l}2.69 \\
(1.55-4.64)\end{array}$ & $<0.001$ & $\begin{array}{l}1.2 \\
(0.53-2.70)\end{array}$ & 0.659 & $\begin{array}{l}0.92 \\
(0.50-1.69)\end{array}$ & 0.781 \\
\hline Dyslipidaemia & $\begin{array}{l}2.14 \\
(1.32-3.49)\end{array}$ & 0.002 & $\begin{array}{l}1.6 \\
(1.05-2.42)\end{array}$ & 0.028 & $\begin{array}{l}1.745 \\
(0.94-3.24)\end{array}$ & 0.075 & $\begin{array}{l}2.68 \\
(1.50-4.78)\end{array}$ & 0.001 \\
\hline
\end{tabular}

DM, diabetes mellitus; Htn., Hypertension; NAFLD, non-alcoholic fatty liver disease

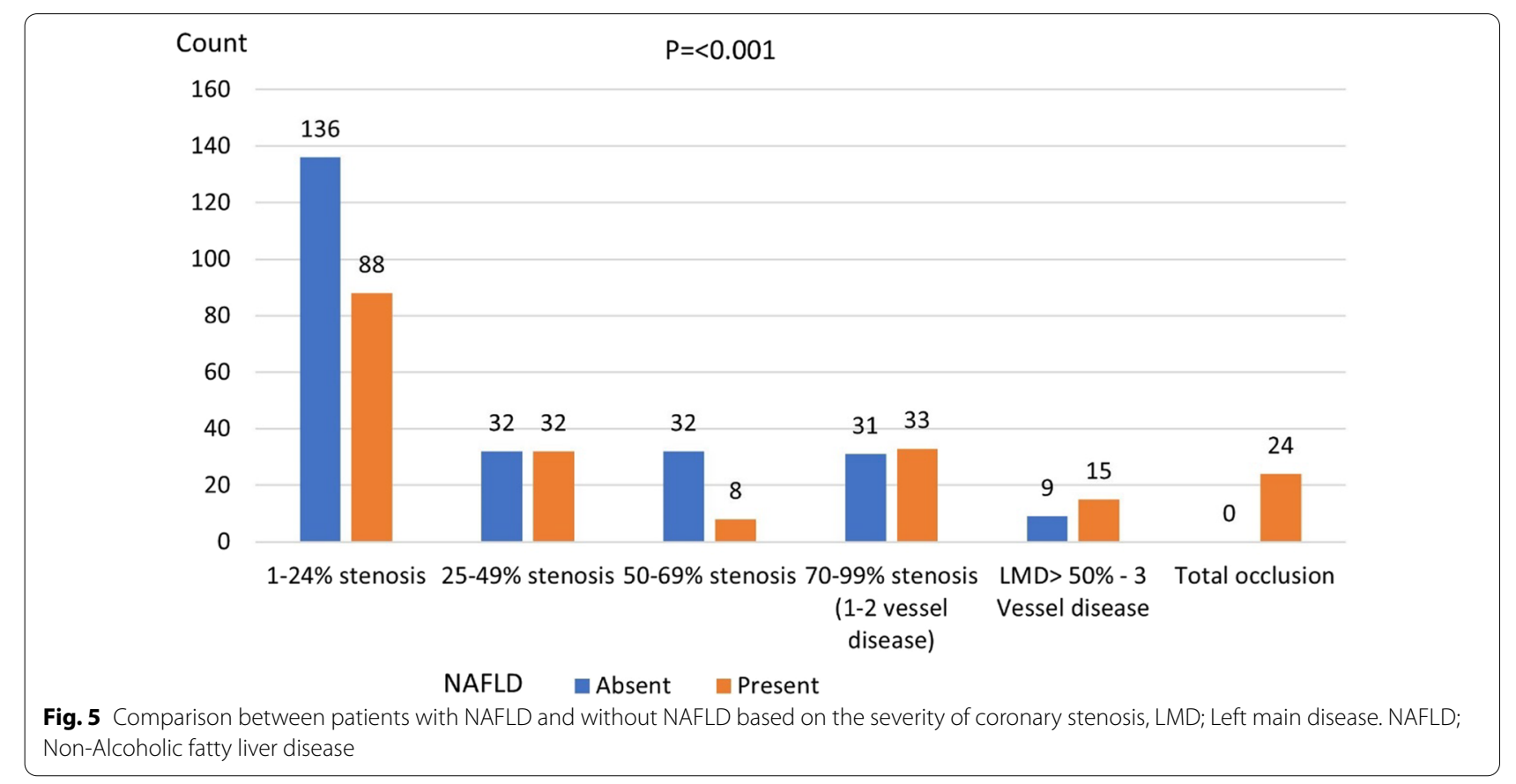

enhanced for the diagnostic purpose for NAFLD or CAD, respectively, as it confers a one-stop-shop for the diagnosis of both diseases, respectively, with adequate diagnostic accuracy $[10,23]$.

In the current study, based on bivariate analysis, CAD was significantly associated with older age, male gender, NAFLD, hypertension, diabetes mellitus, smoking and dyslipidemia. However, the multivariate logistic regression analysis including the abovementioned factors, showed age, smoking and NAFLD to be the only independent risk factors for CAD.

The absence of a significant association between DM, hypertension, and dyslipidemia in the multivariate model was unanticipated, owing to the well-established relation between CAD and those major modifiable risk factors [24-26]. Our findings could be attributed to us being unaware of the duration and degree of control of those risk factors in our patients. 
In our study, patients with NAFLD were almost four times more likely to have CAD. Moreover, high-risk plaque features were more frequently encountered in those patients. This can be explained theoretically by the fact that NAFLD represents an interplay among all other risk factors [7]. This finding is affirmative to Puchner et al., 2015 study, which retrospectively analysed data from the Rule Out Myocardial Infarction by Cardiac Computed Tomography II (ROMICAT II) CAD arm in patients with acute coronary syndrome [15]. However, our study was prospectively conducted and included patients with suspected acute and chronic coronary syndromes.

Ismael et al., 2020 found a similar association between NAFLD and CAD where they used the coronary angiography for coronary evaluation and FibroScan for the detection of liver steatosis tested on 100 patients [27].

We have found that NAFLD was significantly more common in patients with increased CAD severity. This finding was similar to what Sun \& Lü, 2011 found [28].

We could not detect any significant association between the presence of NAFLD and a calcium score over $100 \mathrm{AU}$. In contrast to Kim et al., 2012 who found a strong relationship between NAFLD and coronary artery calcification [29]. This can be attributed to the lower number of cases in our study who had a coronary calcium score above 100 AU (only $10 \%$ of the whole cohort) and those with high calcium score obscuring segmental interpretation of coronary stenosis were excluded.

\section{Conclusions}

The high prevalence of NAFLD and its strong association with the presence of CAD confers additional independent risk for CAD. NAFLD is significantly associated with advanced high-risk coronary plaque features and a higher grade of coronary stenosis.

\section{Limitations of the study}

The single-centre, cross-sectional design of our study is considered a limitation to corroborate a causal or temporal relationship between NAFLD and the development of CAD. Also, the use of unenhanced CT for diagnosis of NAFLD CT might not be as accurate for detecting mild steatosis [30].

\footnotetext{
Abbreviations

ANOVA: Analysis Of VAriance; AU: Agatston Units; CAD: Coronary Artery Disease; CAD-RADS: Coronary Artery Disease Reporting and Data System; CCTA: Coronary Computed Tomography Angiography; Cl: Confidence Interval; CT: Computed Tomography; DM: Diabetes mellitus; Htn.: Hypertension; HU: Hounsfield Unit; LMD: Left Main Disease; NAFLD: Non-Alcoholic Fatty Liver Disease; OR: Odds Ratio; ROMICAT II: Rule Out Myocardial Infarction by Cardiac Computed Tomography II; SPSS: Statistical Package For Social Science.
}

\section{Acknowledgements}

Not applicable.

\section{Authors' contributions}

All authors have read and approved the manuscript. SS contributed to interpretation of the Coronary CT (Computed Tomography) angiography findings, shared in writing and revision of the methodology. MS contributed to revision of the clinical data, appropriateness for enrolment and shared in writing and revision of the manuscript, statistical analysis, and corresponding author. MM contributed to interpretation of the $\mathrm{CT}$ abdomen findings, shared in writing and revision of the methodology. MG contributed to revision of the scientific data, shared in writing. $\mathrm{HH}$ contributed to interpretation of the Coronary CT angiography findings, shared in writing and revision of the methodology. MR contributed to interpretation of the $\mathrm{CT}$ abdomen findings, shared in writing and revision of the methodology.

\section{Funding}

Self-funding.

\section{Availability of data and materials}

The data underlying this article are available in the digital library of radiology department Cairo University and cannot be shared publicly due to patients' privacy and confidentiality as per hospital policy.

\section{Declarations}

\section{Ethics approval and consent to participate}

The study protocol conformed to the ethical guidelines of the 1975 Declaration of Helsinki and was approved by the Institutional Review Board of Radiology, Cairo University Hospitals. A written informed consent was obtained from all participants before enrolling.

\section{Consent for publication}

Not applicable.

\section{Competing interests}

All authors declare they have no competing interests.

\section{Author details}

${ }^{1}$ Cardiovascular Department, Faculty of Medicine, Cairo University, 12 Al Saraya Street, El Manial, Cairo P.O 11555, Egypt. ${ }^{2}$ Radiology Department, Faculty of Medicine, Cairo University, 12 Al Saraya Street, El Manial, Cairo P.O 11555 , Egypt. ${ }^{3}$ Students' Hospital, Cairo University, 15 Gamal Eldin Afify street, Giza square, Giza P.O 12511, Egypt. ${ }^{4}$ Radiology Department, Students' Hospital, Cairo University, 15 Gamal Eldin Afify street, Giza square, Giza P.O 12511, Egypt.

Received: 16 June 2021 Accepted: 21 October 2021

Published online: 30 October 2021

\section{References}

1. Anstee QM, McPherson S, Day CP (2011) How big a problem is nonalcoholic fatty liver disease? BMJ 343:d3897. https://doi.org/10.1136/bmj. d3897

2. Anstee QM, Targher G, Day CP (2013) Progression of NAFLD to diabetes mellitus, cardiovascular disease or cirrhosis. Nat Rev Gastroenterol Hepatol 10(6):330-344. https://doi.org/10.1038/nrgastro.2013.41

3. Browning JD, Szczepaniak LS, Dobbins R, Nuremberg P, Horton JD, Cohen JC et al (2004) Prevalence of hepatic steatosis in an urban population in the United States: impact of ethnicity. Hepatology 40(6):1387-1395. https://doi.org/10.1002/hep.20466

4. Loomba R, Sanyal AJ (2013) The global NAFLD epidemic. Nat Rev Gastroenterol Hepatol 10:686-690

5. Younossi ZM, Koenig AB, Abdelatif D, Fazel Y, Henry L, Wymer M (2016) Global epidemiology of nonalcoholic fatty liver disease — meta-analytic assessment of prevalence, incidence, and outcomes. Hepatology 64(1):73-84. https://doi.org/10.1002/hep.28431 
6. Masuoka HC, Chalasani N (2013) Nonalcoholic fatty liver disease: an emerging threat to obese and diabetic individuals. Ann NY Acad Sci 1281(1):106-122. https://doi.org/10.1111/nyas.12016

7. Stahl EP, Dhindsa DS, Lee SK, Sandesara PB, Chalasani NP, Sperling LS (2019) Nonalcoholic fatty liver disease and the heart: JACC state-of-theart review. J Am Coll Cardiol 73(8):948-963. https://doi.org/10.1016/j.jacc. 2018.11.050

8. Mahfood Haddad T, Hamdeh S, Kanmanthareddy A, Alla VM (2017) Nonalcoholic fatty liver disease and the risk of clinical cardiovascular events: a systematic review and meta-analysis. Diabetes Metab Syndr 11 (Suppl 1):S209-s216. https://doi.org/10.1016/j.dsx.2016.12.033

9. Ferencik M, Mayrhofer T, Bittner DO, Emami H, Puchner SB, Lu MT et al (2018) Use of high-risk coronary atherosclerotic plaque detection for risk stratification of patients with stable chest pain: a secondary analysis of the PROMISE randomized clinical trial. JAMA Cardiol 3(2):144-152. https:// doi.org/10.1001/jamacardio.2017.4973

10. Li Q, Dhyani M, Grajo JR, Sirlin C, Samir AE (2018) Current status of imaging in nonalcoholic fatty liver disease. World J Hepatol 10(8):530-542. https://doi.org/10.4254/wjh.v10.i8.530

11. Boyce CJ, Pickhardt PJ, Kim DH, Taylor AJ, Winter TC, Bruce RJ et al (2010) Hepatic steatosis (fatty liver disease) in asymptomatic adults identified by unenhanced low-dose CT. Am J Roentgenol 194(3):623-628. https://doi. org/10.2214/AJR.09.2590

12. Park YS, Park SH, Lee SS, Kim DY, Shin YM, Lee W et al (2011) Biopsyproven nonsteatotic liver in adults: estimation of reference range for difference in attenuation between the liver and the spleen at nonenhanced CT. Radiology 258(3):760-766. https://doi.org/10.1148/radiol.10101233

13. Abbara S, Blanke P, Maroules CD, Cheezum M, Choi AD, Han BK et al (2016) SCCT guidelines for the performance and acquisition of coronary computed tomographic angiography: a report of the society of Cardiovascular Computed Tomography Guidelines Committee: Endorsed by the North American Society for Cardiovascular Imaging (NASCI). J Cardiovasc Comput Tomogr 10(6):435-449. https://doi.org/10.1016/j.jcct.2016.10.002

14. Cury RC, Abbara S, Achenbach S, Agatston A, Berman DS, Budoff MJ et al (2016) CAD-RADS(TM) Coronary Artery Disease-Reporting and Data System. An expert consensus document of the Society of Cardiovascular Computed Tomography (SCCT), the American College of Radiology (ACR) and the North American Society for Cardiovascular Imaging (NASCl). Endorsed by the American College of Cardiology. J Cardiovasc Comput Tomogr 10(4):269-281. https://doi.org/10.1016/j.jcct.2016.04.005

15. Puchner SB, Lu MT, Mayrhofer T, Liu T, Pursnani A, Ghoshhajra BB et al (2015) High-risk coronary plaque at coronary CT angiography is associated with nonalcoholic fatty liver disease, independent of coronary plaque and stenosis burden: results from the ROMICAT II trial. Radiology 274(3):693-701. https://doi.org/10.1148/radiol.14140933

16. Tana C, Ballestri S, Ricci F, Di Vincenzo A, Ticinesi A, Gallina S et al (2019) Cardiovascular risk in non-alcoholic fatty liver disease: mechanisms and therapeutic implications. Int J Environ Res Public Health. https://doi.org/ 10.3390/ijerph16173104

17. Kumar R, Priyadarshi RN, Anand U (2020) Non-alcoholic fatty liver disease: growing burden, adverse outcomes and associations. J Clin Transl Hepatol 8(1):76-86. https://doi.org/10.14218/jcth.2019.00051
18. Younossi ZM, Diehl AM, Ong JP (2002) Nonalcoholic fatty liver disease: an agenda for clinical research. Hepatology 35(4):746-752

19. Wong VWS, Hui AY, Tsang SWC, Chan JLY, Tse AML, Chan KF et al (2006) Metabolic and adipokine profile of Chinese patients with nonalcoholic fatty liver disease. Clin Gastroenterol Hepatol 4(9):1154-1161

20. Le MH, Devaki P, Ha NB, Jun DW, Te HS, Cheung RC, Nguyen MH (2017) Prevalence of non-alcoholic fatty liver disease and risk factors for advanced fibrosis and mortality in the United States. PLoS ONE 12(3):e0173499. https://doi.org/10.1371/journal.pone.0173499

21. Younossi Z, Anstee QM, Marietti M, Hardy T, Henry L, Eslam M et al (2018) Global burden of NAFLD and NASH: trends, predictions, risk factors and prevention. Nat Rev Gastroenterol Hepatol 15(1):11-20. https://doi.org/ 10.1038/nrgastro.2017.109

22. Ahmed MH, Noor SK, Bushara SO, Husain NE, Elmadhoun WM, Ginawi IA et al (2017) Non-alcoholic fatty liver disease in Africa and Middle East: an attempt to predict the present and future implications on the healthcare system. Gastroenterol Res 10(5):271-279. https://doi.org/10.14740/ gr913w

23. Lawrence DA, Oliva IB, Israel GM (2012) Detection of hepatic steatosis on contrast-enhanced CT images: diagnostic accuracy of identification of areas of presumed focal fatty sparing. Am J Roentgenol 199(1):44-47. https://doi.org/10.2214/AJR.11.7838

24. Fuchs FD, Whelton PK (2020) High blood pressure and cardiovascular disease. Hypertension 75(2):285-292. https://doi.org/10.1161/HYPER TENSIONAHA.119.14240

25. Lu S, Bao M-Y, Miao S-M, Zhang X, Jia Q-Q, Jing S-Q et al (2019) Prevalence of hypertension, diabetes, and dyslipidemia, and their additive effects on myocardial infarction and stroke: a cross-sectional study in Nanjing, China. Ann Transl Med 7(18):436-436. https://doi.org/10.21037/atm.2019. 09.04

26. Hedayatnia M, Asadi Z, Zare-Feyzabadi R, Yaghooti-Khorasani M, Ghazizadeh H, Ghaffarian-Zirak R et al (2020) Dyslipidemia and cardiovascular disease risk among the MASHAD study population. Lipids Health Dis 19(1):42. https://doi.org/10.1186/s12944-020-01204-y

27. Ismael H, Tag-Adeen M, Abdel-Rady A, Shazly M, Hussein A (2020) Nonalcoholic fatty liver disease as a coronary heart disease severity predictor. Int J Clin Med 11(04):182

28. Sun L, Lü S-Z (2011) Association between non-alcoholic fatty liver disease and coronary artery disease severity. Chin Med J 124(6):867-872

29. Kim D, Choi SY, Park EH, Lee W, Kang JH, Kim W et al (2012) Nonalcoholic fatty liver disease is associated with coronary artery calcification. Hepatology 56(2):605-613. https://doi.org/10.1002/hep.25593

30. Efe D, Aygün F (2014) Assessment of the relationship between nonalcoholic fatty liver disease and CAD using MSCT. Arq Bras Cardiol 102(1):10-18. https://doi.org/10.5935/abc.20130225

\section{Publisher's Note}

Springer Nature remains neutral with regard to jurisdictional claims in published maps and institutional affiliations.

\section{Submit your manuscript to a SpringerOpen ${ }^{\odot}$ journal and benefit from:}

- Convenient online submission

- Rigorous peer review

- Open access: articles freely available online

- High visibility within the field

- Retaining the copyright to your article

Submit your next manuscript at springeropen.com 